\title{
Sulfited tannin capsules: novel stimuli-responsive
}

\section{delivery systems}

Luc Zongo, ${ }^{\mathrm{a}}$ Heiko Lange, $,{ }^{\mathrm{b}, \dagger}, *$ and Claudia Crestini ${ }^{\mathrm{c}, \grave{\dagger}, *}$

a University of Rome 'Tor Vergata', Department of Chemical Science and Technologies, Via della Ricerca Scientifica, 00133 Rome, Italy

b University of Milano-Bicocca, Department of Earth and Environmental Sciences, Piazza della Scienza 1, 20126 Milan, Italy

c University of Venice 'Ca' Foscari', Department of Molecular Science and Nanosystems, Via Torino 155, 30170 Venice Mestre, Italy

$\dagger$ Affiliated with a) via NAST - Nanoscience \& Nanotechnology \& Innovative Instrumentation Center.

* Corresponding authors: heiko.lange@unimib.it

claudia.crestini@unive.it

Number of pages: 7

Number of figures: 6

Number of tables: 3 


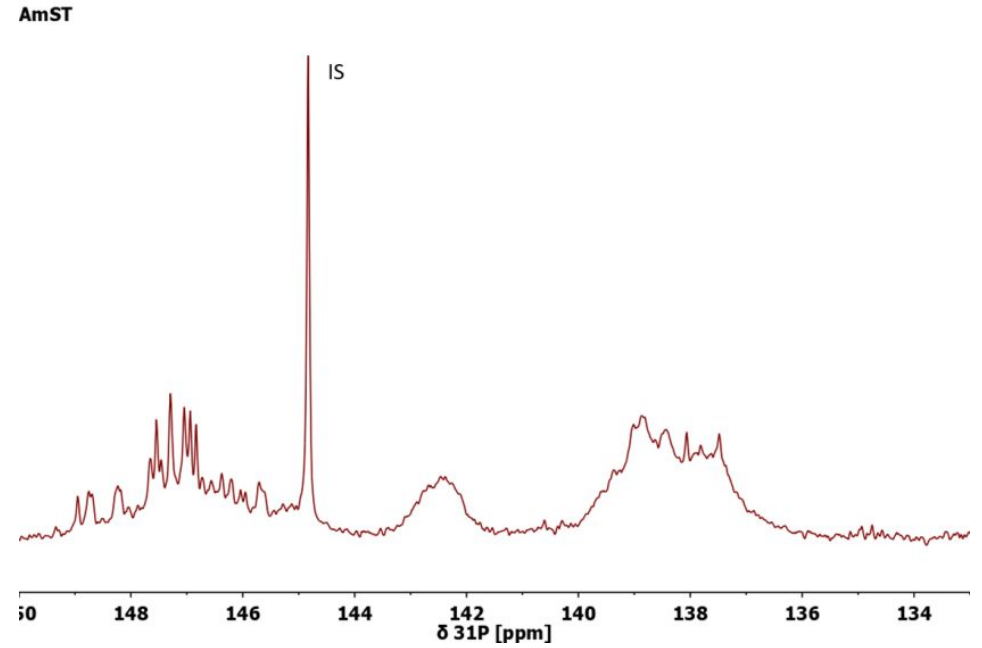

Figure S1: ${ }^{31} \mathrm{P}$ NMR spectrum of phosphitylated $\mathbf{A m S T}$. Sample preparation and quantitative measurement were performed as described in detail before. ${ }^{1-3}$ In brief, approx. $15 \mathrm{mg}$ of tannin were accurately weighed and added to 450 $\mu \mathrm{L}$ of a mixture of pyridine $/ \mathrm{CDCl}_{3}$ (1.6:1). One hundred microliters of the standard solution, prepared using cholesterol at a concentration of $0.1 \mathrm{M}$ in the above-mentioned solvent mixture mixed with $50 \mathrm{mg} / \mathrm{mL}$ of $\mathrm{Cr}$ (III) acetylacetonate as relaxation agent, were added, followed by $50 \mu \mathrm{L}$ of 2-chloro-4,4,5,5-tetramethyl-1,3,2-dioxaphospholane. After 1 hour stirring at room temperature the functionalized mixture was quantitatively transferred to a standard NMR tube for analysis. ${ }^{31} \mathrm{P}$ NMR spectra were recorded on a Bruker $300 \mathrm{MHz}$ spectrometer at $20^{\circ} \mathrm{C}$ using an inverse gated decoupling sequence with a delay of $10 \mathrm{~s}$ between successive pulses. Chemical shifts were expressed in parts per million from $85 \%$ $\mathrm{H}_{3} \mathrm{PO}_{4}$ as an external reference. All chemical shifts reported are relative to the peak for the reaction product of water with Cl-TMDP $132.2 \mathrm{ppm}$ in the used conditions. NMR data were processed with MestreNova (Version 8.1.1, Mestrelab Research).

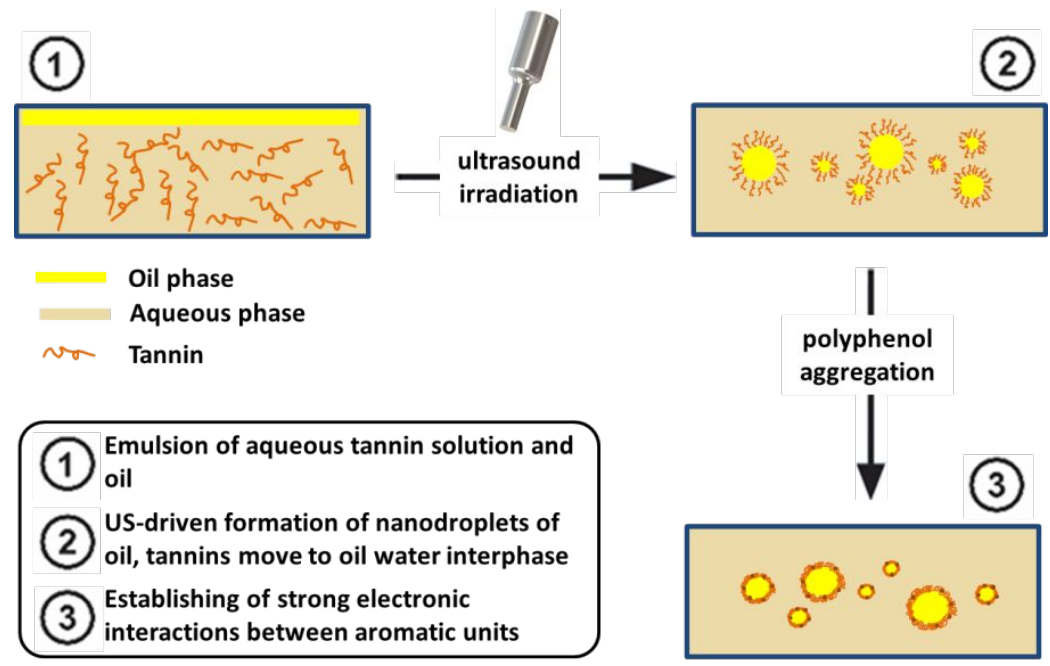

Figure S2: Schematic representation of the ultrasonication-based process used for capsule formation. Details are given in the Experimental Section of the main text. 

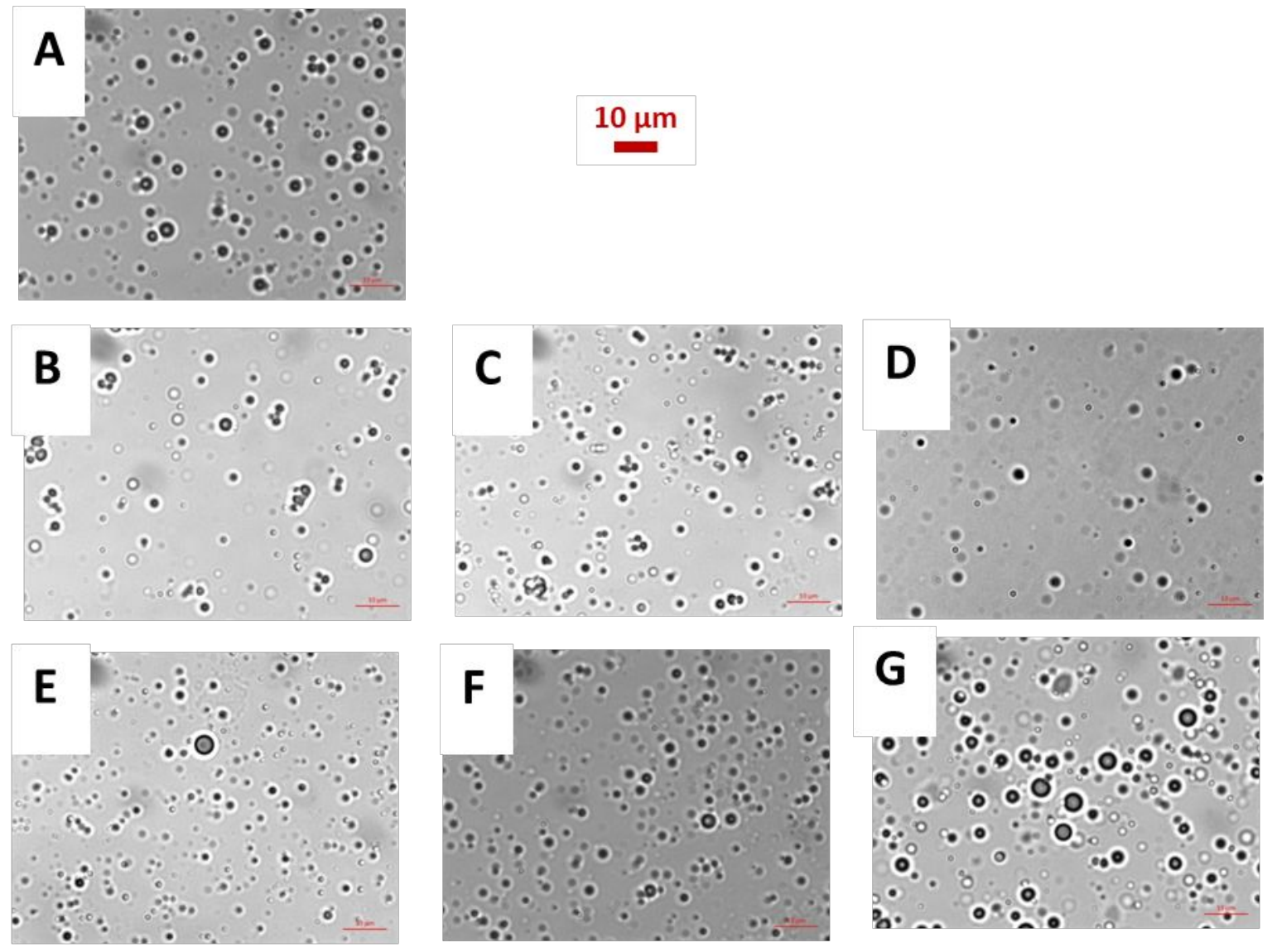
$A=$ Control
$E=A O$
$B=A E-25$
$F=$ MOPS
$C=S D S$
$G=$ Glucose
$\mathrm{D}=\mathrm{NaCl}$

Figure S3: Representative microscope images of solutions of AmST-MCs after incubation in solutions of various surfactants. All pictures represent the same dilution factor for analysis, i.e., 1/100. 


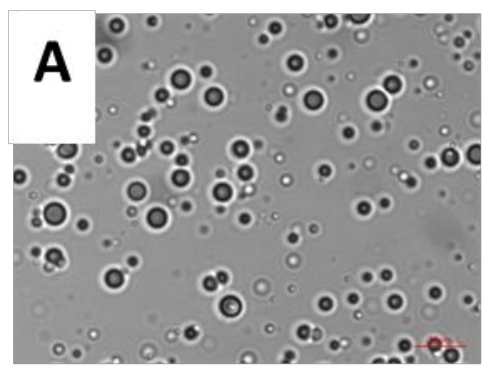

\section{$10 \mu \mathrm{m}$}
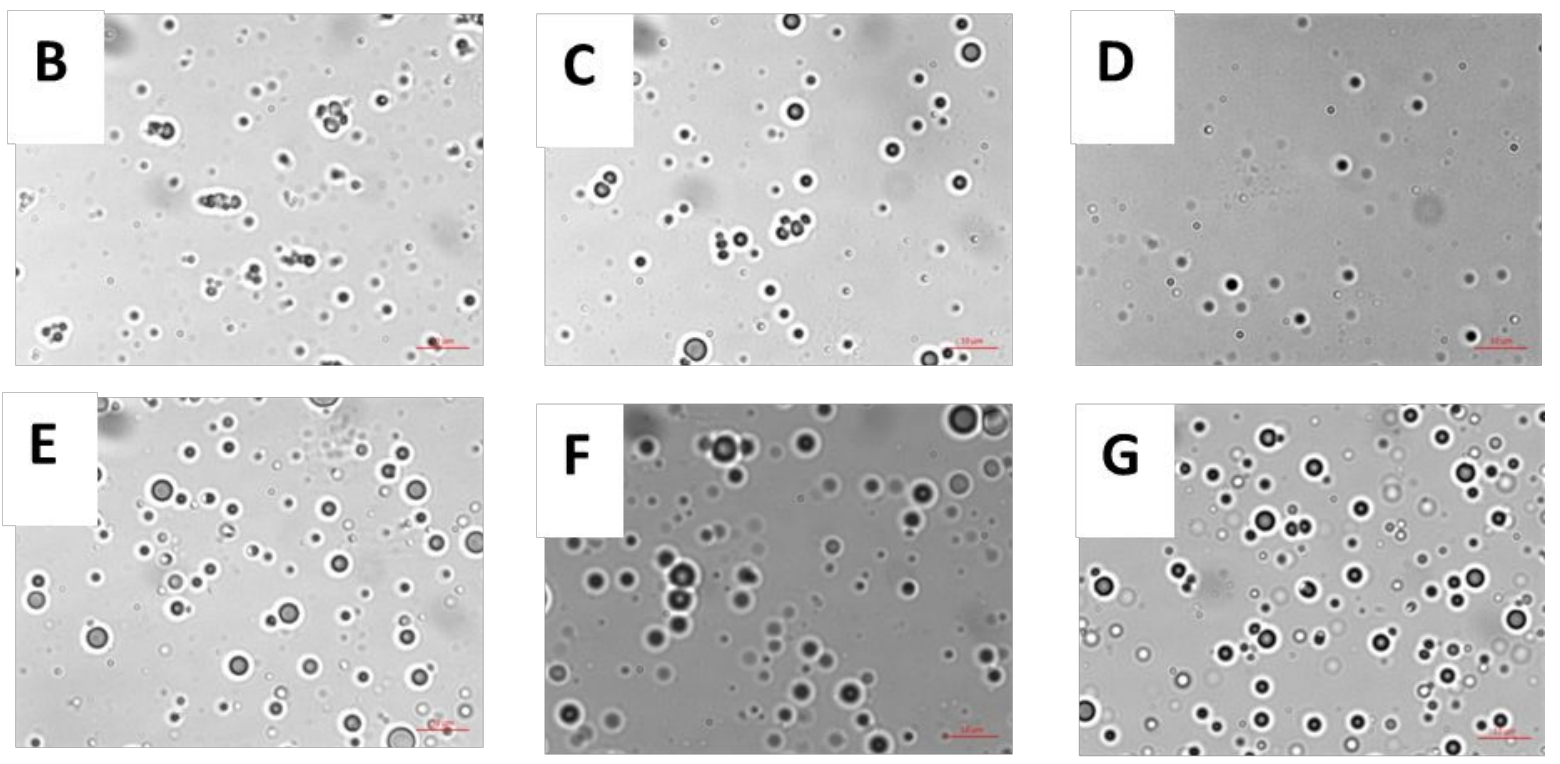
$A=$ Control
$E=A O$
$B=A E-25$
$F=$ MOPS
$C=$ SDS
G = Glucose
$D=\mathrm{NaCl}$

Figure S4: Representative microscope images of solutions of $\boldsymbol{A m T}$-MCs after incubation in solutions of various surfactants. All pictures represent the same dilution factor for analysis, i.e., 1/100. 
Table S1: Assessment of the effect of organic molecules on the disassembly of $\boldsymbol{A m S T - M C s}$ and $\boldsymbol{A m}$ T-MCs.

\begin{tabular}{|c|c|c|c|c|c|c|c|c|c|c|c|}
\hline \multirow[t]{2}{*}{ entry } & \multirow[t]{2}{*}{$\begin{array}{l}\text { org. molecule } \\
\text { solution }\end{array}$} & \multirow[t]{2}{*}{ pH } & \multirow[t]{2}{*}{$\begin{array}{c}\text { conc. } \\
{[\% \mathrm{w} / \mathrm{w}]}\end{array}$} & \multicolumn{2}{|c|}{$\begin{array}{c}\mathbf{A U L} \varnothing \\
{[\mu \mathrm{m}]}\end{array}$} & \multicolumn{2}{|c|}{ PDI } & \multicolumn{2}{|c|}{$\begin{array}{c}\text { \# MCs } \\
{\left[10^{12} \mathrm{MCs} / \mathrm{mL}\right]}\end{array}$} & \multicolumn{2}{|l|}{$\begin{array}{l}\text { stability } \\
\text { index }\end{array}$} \\
\hline & & & & $\underline{A m S T}$ & $A m \mathrm{~T}$ & $A m \mathrm{ST}$ & $A m \mathrm{~T}$ & $A m S T$ & $A m \mathrm{~T}$ & $A m \mathrm{ST}$ & $A m \mathrm{~T}$ \\
\hline 1 & $\mathrm{H}_{2} \mathrm{O}$ [Ref.] & $\sim 7$ & - & 1.44 & 1.28 & 0.5 & 0.5 & 6.97 & 6.64 & 1.00 & 1.00 \\
\hline 2 & $\mathbf{B S A}^{\mathrm{a}}$ & $\sim 8$ & 6 & 1.32 & 1.40 & 0.5 & 0.5 & 6.62 & 5.78 & 0.95 & 0.87 \\
\hline 3 & $\mathrm{PBM}-1^{\mathrm{b}}$ & $\sim 8$ & 6 & 1.35 & 1.51 & 0.5 & 0.4 & 5.84 & 4.18 & 0.84 & 0.63 \\
\hline 4 & PBM-2c & $\sim 6$ & 6 & 1.54 & 1.37 & 0.5 & 0.5 & 5.92 & 5.11 & 0.85 & 0.77 \\
\hline
\end{tabular}

a: bovine serum albumin

b: physiological background mixture-1 in phosphate buffer system

c: physiological background mixture-2 in acetate buffer system

Table S2: Assessment of the disassembly of $\boldsymbol{A m}$ T MCs and AmST-MCs in organic solvents

\begin{tabular}{|c|c|c|c|c|c|c|c|c|c|c|c|c|c|}
\hline \multirow[t]{2}{*}{ entry } & \multirow[t]{2}{*}{$\begin{array}{l}\text { organic } \\
\text { solvent }\end{array}$} & \multicolumn{2}{|c|}{$\begin{array}{c}\text { AUL Ø } \\
{[\mu \mathrm{m}]}\end{array}$} & \multicolumn{2}{|c|}{ PDI } & \multicolumn{2}{|c|}{$\begin{array}{c}\text { \# TMCs } \\
{\left[10^{12} \text { TMCs } / \mathrm{mL}\right]}\end{array}$} & \multicolumn{2}{|c|}{ stability index } & \multicolumn{4}{|c|}{ identified oil component $^{\mathrm{a}}$} \\
\hline & & $A m \mathrm{ST}$ & $A m \mathrm{~T}$ & $A m \mathrm{ST}$ & $A m \mathrm{~T}$ & $A m \mathrm{ST}$ & $A m \mathrm{~T}$ & $A m \mathrm{ST}$ & $A m \mathrm{~T}$ & $\mathbf{A}$ & B & $\mathrm{C}$ & D \\
\hline 1 & reference & 1.44 & 1.28 & 0.5 & 0.5 & 6.97 & 6.64 & 1.00 & 1.00 & - & - & - & - \\
\hline 2 & toluene & 1.23 & 1.24 & 0.4 & 0.4 & 2.30 & 2.90 & 0.35 & 0.39 & 8.55 & 1.10 & 58.64 & 6.46 \\
\hline 3 & hexane & 1.40 & 1.35 & 0.6 & 0.6 & 4.95 & 3.67 & 0.56 & 0.51 & 10.85 & 1.13 & 61.83 & 7.56 \\
\hline 4 & ethanol & - & - & - & - & - & - & - & - & 2.45 & 5.96 & 26.32 & 13.47 \\
\hline
\end{tabular}

a: Area \% of the GC-MS data obtained from the oily phase and the concentrated organic solvents from the dialysis: palmitic acid (A), linoleic acid (B), oleic acid (C) and Stearic acid (D).

Table S3: Buffer systems at different $\mathrm{pH}$, salinity and molarity

\begin{tabular}{clccc}
\hline entry & buffer system & $\begin{array}{c}\text { pH } \\
\text { salinity } \\
\text { [ppt] }\end{array}$ & $\begin{array}{c}\text { molarity } \\
\text { [M] }\end{array}$ \\
\hline 1 & $\mathrm{KCl} / \mathrm{HCl}$ & 1 & 4.8 & 0.1 \\
2 & $\mathrm{KCl} / \mathrm{HCl}$ & 2 & 5.8 & 0.1 \\
3 & $\mathrm{CH} \mathrm{COOH}_{3} / \mathrm{CH}_{3} \mathrm{COONa}$ & 3 & 6.0 & 0.1 \\
4 & $\mathrm{CH}_{3} \mathrm{COOH} / \mathrm{CH}_{3} \mathrm{COONa}$ & 4 & 6.3 & 0.1 \\
5 & $\mathrm{CH}_{3} \mathrm{COOH} / \mathrm{CH}_{3} \mathrm{COONa}$ & 5 & 7.4 & 0.1 \\
6 & $\mathrm{CH}_{3} \mathrm{COOH} / \mathrm{CH}_{3} \mathrm{COONa}_{2}$ & 6 & 8.1 & 0.1 \\
7 & $\mathrm{NaH}_{2} \mathrm{PO}_{4} / \mathrm{Na}_{2} \mathrm{HPO}_{4}$ & 7 & 12.9 & 0.1 \\
8 & $\mathrm{NaH}_{2} \mathrm{PO}_{4} / \mathrm{Na}_{2} \mathrm{HPO}_{4}$ & 7 & 2.6 & 0.02 \\
9 & $\mathrm{NaH}_{2} \mathrm{PO}_{4} / \mathrm{Na}_{2} \mathrm{HPO}_{4}$ & 8 & 12.1 & 0.1 \\
10 & $\mathrm{NaHCO}_{3} / \mathrm{Na}_{2} \mathrm{CO}_{3}$ & 9 & 8.6 & 0.1 \\
11 & $\mathrm{NaHCO}_{3} / \mathrm{Na}_{2} \mathrm{CO}_{3}$ & 10 & 9.5 & 0.1 \\
12 & $\mathrm{NaHCO}_{3} / \mathrm{Na}_{2} \mathrm{CO}_{3}$ & 11 & 10.4 & 0.1 \\
13 & $\mathrm{Na}_{2} \mathrm{HPO}_{4} / \mathrm{Na}_{3} \mathrm{PO}_{4}$ & 12 & 9.1 & 0.1 \\
14 & $\mathrm{Na}_{2} \mathrm{HPO}_{4} / \mathrm{Na}_{3} \mathrm{PO}_{4}$ & 12 & 4.6 & 0.05 \\
\hline
\end{tabular}




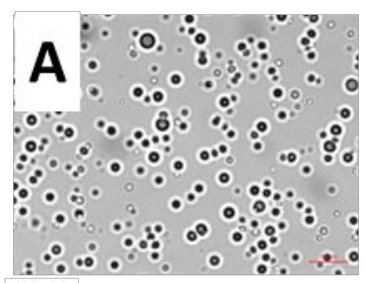

\section{$10 \mu \mathrm{m}$}
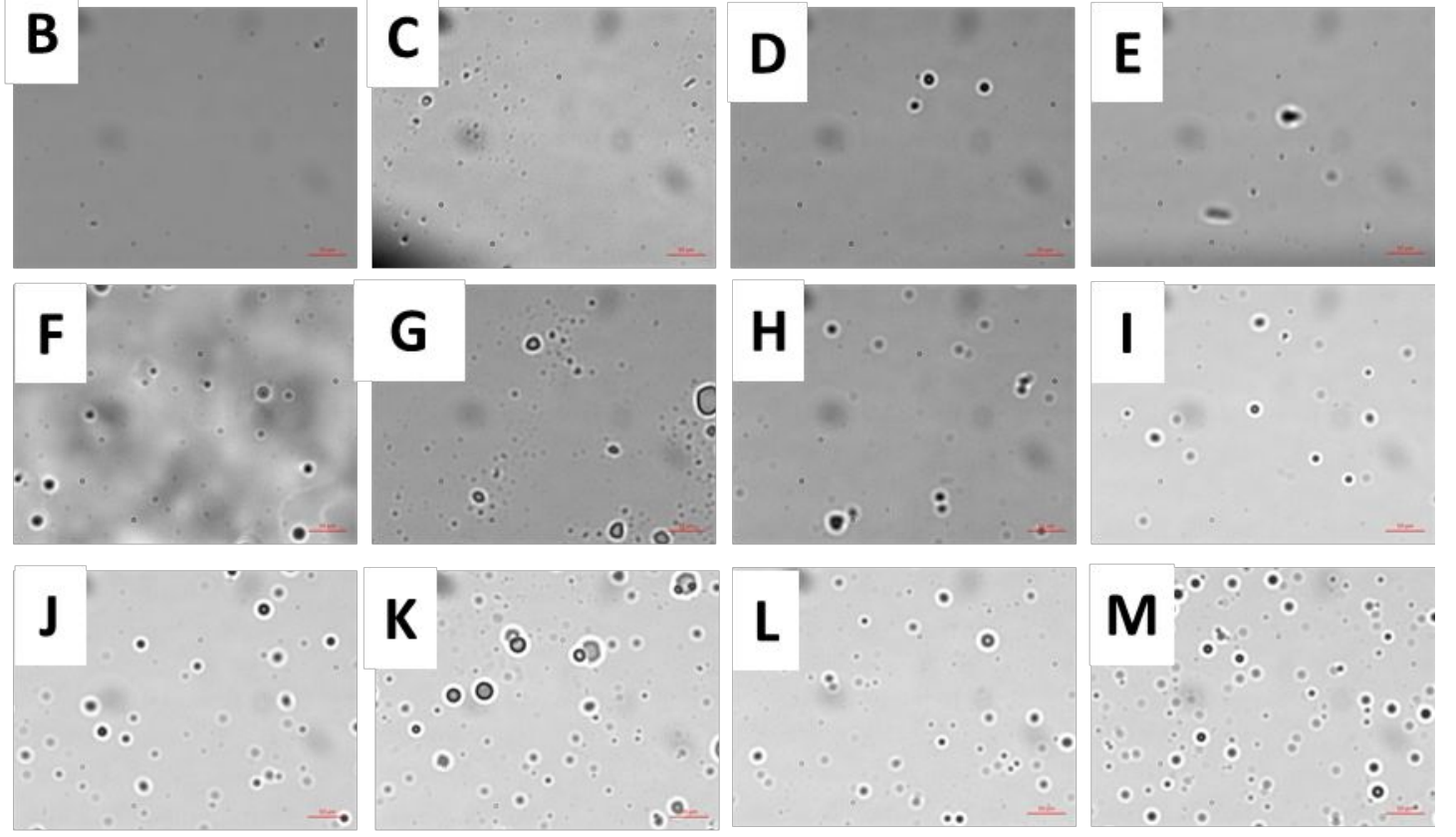

\begin{tabular}{|c|c|c|}
\hline$A=$ Control & $F=p H 5$ & $\mathrm{~J}=\mathrm{pH} 9$ \\
\hline$B=p H 1$ & $\mathrm{G}=\mathrm{pH} 6$ & $\mathrm{~K}=\mathrm{pH} 10$ \\
\hline$C=p H 2$ & $\mathrm{H}=\mathrm{pH} 7$ & $\mathrm{~L}=\mathrm{pH} 11$ \\
\hline$D=p H 3$ & $\mathrm{I}=\mathrm{pH} 8$ & $\mathrm{M}=\mathrm{pH} 12$ \\
\hline$E=p H 4$ & & \\
\hline
\end{tabular}

Figure S5: Representative microscope images of solutions of $\mathbf{A m S T - M C s}$ after incubation in solutions of various $\mathrm{pH}$. All pictures represent the same dilution factor for analysis, i.e., 1/100. 

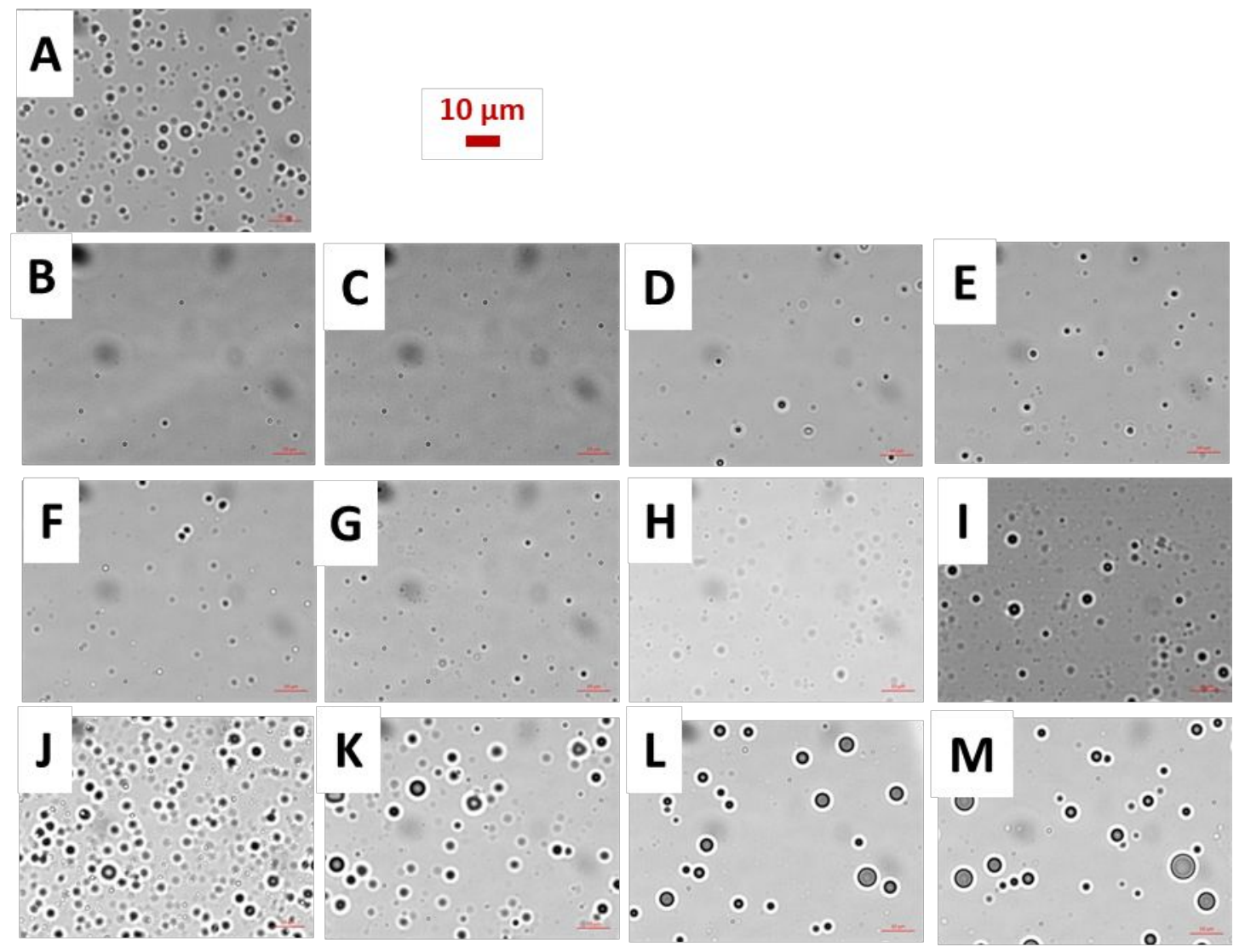

\begin{tabular}{|c|c|c|}
\hline$A=$ Control & $F=p H 5$ & $\mathrm{~J}=\mathrm{pH} 9$ \\
\hline$B=p H 1$ & $G=p H ~ 6$ & $\mathrm{~K}=\mathrm{pH} 10$ \\
\hline$C=p H 2$ & $\mathrm{H}=\mathrm{pH} 7$ & $L=p H 11$ \\
\hline$D=p H 3$ & $\mathrm{I}=\mathrm{pH} 8$ & $M=p H 12$ \\
\hline$E=p H 4$ & & \\
\hline
\end{tabular}

Figure S6: Representative microscope images of solutions of $\boldsymbol{A m T}$ T-MCs after incubation in solutions of various $\mathrm{pH}$. All pictures represent the same dilution factor for analysis, i.e., 1/100.

\section{References}

(1) Melone, F.; Saladino, R.; Crestini, C. Tannin Structural Elucidation and Quantitative 31P NMR Analysis. 1. Model Compounds. J. Agric. Food Chem. 2013, 61 (39), 9307-9315. DOI: 10.1021/jf401477c.

(2) Melone, F.; Saladino, R.; Crestini, C. Tannin Structural Elucidation and Quantitative 31P NMR Analysis. 2. Hydrolyzable Tannins and Proanthocyanidins. J. Agric. Food Chem. 2013, 61 (39), 9316-9324. httpsDOI: $10.1021 / \mathrm{jf} 401664 \mathrm{a}$.

(3) Crestini, C.; Lange, H.; Bianchetti, G. Detailed Chemical Composition of Condensed Tannins via Quantitative 31P NMR and HSQC Analyses: Acacia Catechu, Schinopsis Balansae, and Acacia Mearnsii. J. Nat. Prod. 2016, 79 (9), 2287-2295. DOI: 10.1021/acs.jnatprod.6b00380. 